INPLASY

PROTOCOL

To cite: Cui et al. Efficacy of

transcranial magnetic

stimulation for reducing

suicidal ideation in depression:

a meta-analysis. Inplasy

protocol 202180065. doi:

10.37766/inplasy2021.8.0065

Received: 16 August 2021

Published: 16 August 2021

Corresponding author:

Yanan Cui

527381828@qq.com

Author Affiliation:

Anhui Medical University

Support: National Natural

Science F.

Review Stage at time of this submission: Piloting of the study selection process.

Conflicts of interest:

None declared.

\section{Efficacy of transcranial magnetic stimulation for reducing suicidal ideation in depression: a meta- analysis}

\author{
Cui, Y1; Li, X2; Fang, H³; Yu, F².
}

Review question / Objective: The aim of this meta-analysis of randomized controlled trials is to evaluate the efficacy and safety of adjunctive transcranial magnetic stimulation intervention for suicidal ideation of depression. Whether could suicidal ideation of depression patients benefit from transcranial magnetic stimulation treatment comparing with placebos?

Eligibility criteria: The selected studies were those that met the following eligibility criteria: (1) randomized controlled trials published in English or Chinese; (2) the age of participants ranged from 13- to 80-years-old; (3) the study group was treated with TMS or a physical intervention with a definite treatment plan, including a different sequence and frequency of neurophysical stimulation; (4) the control group had no restrictions in the treatment they received, including conventional treatment, placebo treatment, and waiting for treatment; and (5) the evaluation results were of suicidal ideation and suicidal behavior.

INPLASY registration number: This protocol was registered with the International Platform of Registered Systematic Review and Meta-Analysis Protocols (INPLASY) on 16 August 2021 and was last updated on 16 August 2021 (registration number INPLASY202180065).

\section{INTRODUCTION}

Review question / Objective: The aim of this meta-analysis of randomized controlled trials is to evaluate the efficacy and safety of adjunctive transcranial magnetic stimulation intervention for suicidal ideation of depression. Whether could suicidal ideation of depression patients benefit from transcranial magnetic stimulation treatment comparing with placebos?

Condition being studied: Major depressive disorder (MDD) is a serious, worldwide mental issue, influencing millions of individuals. More than $\mathbf{5 0 \%}$ of Chinese patients with MDD have suicidal ideation. 
Suicide is not only a major health problem but also a social problem. According to global data released by the World Health Organization in 2012, more than 800, 000 people die by suicide every year, accounting for $1.4 \%$ of the world's death toll and making it the 15th leading cause of death. The lifetime prevalence of suicide ideation is approximately $9.2 \%$ on a global scale. Suicidal ideation is defined as thinking about, considering, or planning suicide. Accessible psychological and pharmacological interventions have meant that advancements have been made in reducing suicide; however, these are not without side effects, which influences their effectiveness and may further negatively affect those already at high risk of suicide. Hence inventive treatment procedures to prevent suicide, for use alongside existing treatments, are fundamentally required.

\section{METHODS}

Participant or population: Suicidal ideation in depression.

Intervention: Transcranial magnetic stimulation was the main intervention.

Comparator: The control group had no restrictions in the treatment they received, including conventional treatment, placebo treatment, and waiting for treatment.

Study designs to be included: Randomized clinical trials will be included.

Eligibility criteria: The selected studies were those that met the following eligibility criteria: (1) randomized controlled trials published in English or Chinese; (2) the age of participants ranged from 13- to 80years-old; (3) the study group was treated with TMS or a physical intervention with a definite treatment plan, including a different sequence and frequency of neurophysical stimulation; (4) the control group had no restrictions in the treatment they received, including conventional treatment, placebo treatment, and waiting for treatment; and (5) the evaluation results were of suicidal ideation and suicidal behavior.
Information sources: PubMed, Web of Science, CBMdisc, WanFang, Chongqing VIP, and CNKI databases.

Main outcome(s): Mean change in suicide score; Measures of effect: SMD.

Additional outcome(s): Mean change in depression score; Measures of effect: SMD.

Quality assessment / Risk of bias analysis: Cochrane risk of bias assessment was used to evaluate the study quality according to the following criteria: random sequence generation, allocation concealment, blinding, incomplete outcome data, selective reporting, and other sources of bias. Each area was ranked for high, low, or unknown bias risk. We also calculated the Jadad score for each of the included studies. In calculating the Jadad score, each study was evaluated according to the quality of randomization, blinding procedures, and description of withdrawals and dropouts. Jadad scores ranged from 0 to 5 , with trials scoring 3 or greater considered good quality trials.

Strategy of data synthesis: Standard Mean Difference (SMD) for random effects models will be used. Between-study heterogeneity will be assessedusing the $x 2$ (Cochran Q) and 12 statistics. Results will be assessed using forest plots and presented as SMD for the main outcome and secondary outcomes. An influence analysis will be performed to as certain the results of the meta-analysis by excluding each of the individual studies. Publication bias will be assessed by a funnel plot for meta-analysis and quantified by the Egger method. Statistical analysis will be conducted using STATA software for Mac v15.1

Subgroup analysis: Subgroup analysis showed that age, type of TMS, frequency of intervention, and stimulation threshold altered the TMS efficacy.

Sensitivity analysis: An influence analysis will be performed to as certain the results of the meta-analysis by excluding each of the individual studies. 
Country(ies) involved: China.

Keywords: transcranial magnetic stimulation; suicidal ideation; metaanalysis; depression; TMS.

Contributions of each author:

Author 1 - Yanan Cui.

Email: 527381828@qq.com

Author 2 - Xiaoming Li.

Email: psyxiaoming@126.com

Author 3 - Haijian Fang.

Email: 2528498501@qq.com

Author 4 - Fengqiong Yu.

Email: yufengqiong@ahmu.edu.cn 